\title{
REVISIÓN HISTÓRICA DE LAS ESPECIES DE BIVALVOS CITADAS EN EL JURÁSICO DE LA CORDILLERA IBÉRICA, ESPANA
}

\author{
Graciela DELVENE 1.2
}

\author{
Dpto. Ciencias de la Tierra (Paleontología). Universidad de \\ Zaragoza. 50)(09 Zaragozal. gdelvene@posta.unizar.es \\ 2 Museo Geominero (IGME). C/. Ríos Rosas. 23. 280(0)3 \\ Madrid. g.delvene@igme.es
}

\begin{abstract}
Delvene, G. 2002. Revisión histórica de las especies de bivalvos citadas en el Jurásico de la Cordillera Ibérica, España. [Historical revision about Jurassic bivalves in the Iberian Range, Spain.] Revista Española de Paleontología, 17(2), 199-210. ISSN 0213-6937.
\end{abstract}

\begin{abstract}
A revision of previous work on Jurassic bivalves from the Iberian Range shows the scarcity of data, especially with respect to the Middle and Upper Jurassic. This is partly due to the poor preservation, partly due to the low abundance of this group in Middle and Upper Jurasic rocks in the Iberian Range as compared to the Lower Jurassic bivalves. A review is presented of authors who have studied, reported or figured Jurassic species of bivalves. Moreover, Middle and Upper Jurassic bivalves figured by classical authors are compared with material collected from the Aragonian Branch of the Iberian Range that has been recently studied.
\end{abstract}

Keywords: Bivalves, Jurassic, XVIII-XXI centuries, Iberian Range, Spain.

\section{RESUMEN}

La revisión de los trabajos sobre bivalvos del Jurásico de la Cordillera Ibérica revela la gran escasez de datos referidos especialmente a los materiales del Jurásico Medio y Superior. Ello se debe, por un lado, al deficiente estado de conservación que normalmente presentan los fósiles y por otro, a la baja abundancia de este grupo en materiales de edad Jurásico Medio-Superior en la Cordillera Ibérica, en comparación con los bivalvos del Jurásico Inferior. En este trabajo se hace una revisión de los autores que han estudiado, citaclo o fíguraclo especies de bivalvos del Jurásico. Además, se compara el material del Jurásico Medio y Superior fïgurado por autores clásicos con la colección procedente de la Rama Aragonesa de la Cordillera Ibérica recientemente estudiada.

Palabras clave: Bivalvos, Jurásico, siglos XVIII-XXI, Cordillera Ibérica, España.

\section{INTRODUCCIÓN}

Los trabajos sobre moluscos bi valvos del Jurásico Medio y Superior de la Cordillera Ibérica son bastante escasos. Muchos de los trabajos a los que se va a hacer referencia son estudios globales de tipo regional en los que los autores citan listas de todos los grupos fósiles entre los que incluyen, normalmente, los bivalvos. Se suele mencionar la edad a la que corresponden y la localidad a la que pertenecen. En este trabajo se quiere mostrar las especies citadas en el Jurásico de la Cordillera Ibérica desde el siglo XVII hasta la actualidad y comparar el registro que cada autor menciona con las especies encontradas en los materiales de edad Calloviense-Kimmeridgiense de la Rama Aragonesa de la
Cordillera Ibérica, que han sido recientemente descritas (Delvene, 2001) y al que haremos referencia a lo largo de esta revisión. El análisis taxonómico que se realizó con más de 3.000 ejemplares de bivalvos dio como resultado la descripción de 83 especies, pertenecientes a 46 géneros y subgéneros de las subclases Palaeotaxodonta, Pteriomorphia, Is ofilibranchia, Palaeoheterodonta, Heterodonta y Anomalodesmata.

\section{SIGLO XVIII}

La obra de Torrubia (1754): Aparato para la Historia Natural, constituye la primera referencia paleontológica sobre la Cordillera Ibérica en España (Pelayo, 1994), y 
contiene las primeras citas y figuraciones de bivalvos (láminas: I- II, IV-VIII y X). Su importancia en Europa hizo que fuera traducida al alemán en 1773. Recientemente ha sido reeditada en Madrid por UEI de Paleontología e Instituto de Geología Económica de Madrid (1994) con motivo de las X Jornadas de Paleontología. El material español de bivalvos estudiado por Torrubia proviene del Lías del sector de Molina de Aragón (ver Bernad, 1999a).

\section{SIGLO XIX}

Verneuil y Collomb (1852) en su obra Itineraire geognostique dans le SE de l'Espagne tratan de los materiales de todas las edades geológicas. Sobre los materiales pertenecientes al Jurásico, indican las secciones óptimas. Respecto a los fósiles, indican una lista en la que mencionan géneros y especies de los fósiles jurásicos del este y sureste de España, localidad donde han sido hallados y edades en las que las mismas especies se han encontrado en Francia. Los autores no indican la edad precisa de los ejemplares, limitándose a mostrar su equivalencia con la escala cronoestratigráfica estándar en Europa. De este listado, 26 especies corresponden a bivalvos y 4 de ellas se han figurado (lám. 3, figs. 4-7). En el Apéndice final se indican las especies coincidentes en el intervalo CallovienseKimmeridgiense de la Rama Aragonesa de la Cordillera Ibérica.

Ezquerra $(1850,1854,1857)$ hace una revisión de los trabajos contemporáneos citando particularmente los de Verneuil y Collomb. Realiza una descripción detallada de todas las edades geológicas, donde menciona los minerales más importantes y forma un pequeño catálogo de especies que abarca todos los grupos fósiles que encuentra. En los "terrenos jurásicos y oolíticos, con agregación de los liásicos", menciona 36 especies de bivalvos que él incluye en la clase Acéfalos y en los órdenes Ortoconquios y Pleuroconquios (ver Apéndice).

Figura 1. Se muestran los ejemplares del Jurásico Medio y Superior figurados por Mallada (1879, 1881, 1884, 1885) y Verneuil y Collomb (1852). Las siglas MPZ corresponden al Museo Paleontológico de la Universidad de Zaragoza donde el material estudiado se ha depositado. a: Lima proboscidea J. Sowerby, reproducción de la figura original de Mallada (1879, 1885, lám. 31, fig. 2); x 0,96 respecto a la escala del autor. b: Lima proboscidea J. Sowerby, reproducción de la figura original de Mallada $(1879,1885$, lám. 31, fig. 1); x 0,96 respecto a la escala del autor. c: Ctenostreon proboscideum (J. Sowerby), valva derecha de ejemplar articulado. Fm. Yátova (Ventas de San Pedro, Oliete, prov. Teruel); x 0,68. MPZ 97/48. d: Mytilus bipartitus (J. Sowerby), reproducción de la figura original de Mallada (1884, 1885, lám. 43, fig. 7); x 0,94 respecto a la escala del autor. e: Modiolus (Modiolus) bipartitus J. Sowerby, vista izquierda de ejemplar articulado. Fm. Yátova (Barranco de las Estacas, Ariño, prov. Teruel); x 1,6. MPZ 00/2374. f: Pholadomya acuminata Hartmann in Zieten, reproducción de la figura original de Mallada (1881, 1885, lám. 30, fig. 6); manteniendo la escala del autor. g: Pholadomya acuminata Hartmann in Zieten, reproducción de la figura original de Mallada $(1881,1885$, lám. 30, fig. 7); manteniendo la escala del autor. h: Pholadomya acuminata Hartmann in Zieten, vista izquierda de molde interno articulado. Fm. Loriguilla (La Cañada de Verich, prov. Teruel); x 1,4. MPZ 00/3076. i: Pholadomya acuminata Hartmann in Zieten, vista izquierda de molde interno articulado. Fm. Sot de Chera (Aguilón, prov. Zaragoza); x 1,3. MPZ 00/3077. j: Pholadomya trapezina (Buvignier), reproducción de la figura original de Mallada $(1881,1885$, lám. 30B, fig. 1); manteniendo la escala del autor. k: Pholadomya trapezina Buvignier, reproducción de la figura original de Verneuil y Collomb (1852, lám. 3, fig. 7); manteniendo la escala del autor. l: Goniomya (Goniomya) trapezicostata (Pusch), vista izquierda de molde interno articulado. Fm. Yátova (Barranco de las Estacas, Ariño, prov. Teruel); x 1. MPZ 00/3075.

Middle and Upper Jurassic specimens figured by Mallada (1879, 1881, 1884, 1885) and Verneuil and Collomb (1852). MPZ letters correspond to "Museo Paleontológico de la Universidad de Zaragoza" where studied fossils are housed. a: Lima proboscidea J. Sowerby, reproduced from the Mallada's original figure (1879, 1885, pl. 31, fig. 2); $x 0.96$ respect to the author's scale. $b$ : Lima proboscidea J. Sowerby, reproduced from the Mallada's original figure $(1879,1885, \mathrm{pl}$. 31, fig. 1); x 0.96 respect to the author's scale. : Ctenostreon proboscideum (J. Sowerby), right view of articuled specimen. Yátova Fm (Ventas de San Pedro, Oliete, prov. Teruel); x 0.68. MPZ 97/48. d: Mytilus bipartitus (J. Sowerby), reproduced from the Mallada's original figure (1884, 1885, pl. 43, fig. 7); $x$ 0.94 respect to the author's scale. $e$ : Modiolus (Modiolus) bipartitus $J$. Sowerby, left view of articuled specimen. Yátova Fm (Barranco de las Estacas, Ariño, prov. Teruel); $x$ 1,6. MPZ 00/2374. $f$ : Pholadomya acuminata Hartmann in Zieten, reproduced from the Mallada's original figure (1881, 1885, pl. 30, fig. 6); the same author's scale. g: Pholadomya acuminata Hartmann in Zieten, reproduced from the Mallada's original figure (1881, 1885, lám. 30, fig. 7); the same author's scale. h: Pholadomya acuminata Hartmann in Zieten, composite mould of articuled specimen, left valve view. Loriguilla Fm (La Cañada de Verich, prov. Teruel); x 1,4. MPZ 00/3076. $i$ : Pholadomya acuminata Hartmann in Zieten, composite mould of articuled specimen, left valve view. Sot de Chera Fm (Aguilón, prov. Zaragoza); $x$ 1,3. MPZ 00/3077. $j$ : Pholadomya trapezina (Buvignier), reproduced from the Mallada's original figure $(1881,1885$, pl. 30B, fig. 1); the same author's scale. $\mathrm{k}$ : Pholadomya trapezina Buvignier, reproduced from the Verneuil y Collomb's figure (1852, pl. 3, fig. 7); the same author's scale. l: Goniomya (Goniomya) trapezicostata (Pusch), composite mould of articuled specimen, left valve view. Yátova Fm (Barranco de las Estacas, Ariño, prov. Teruel); $x$ 1. MPZ 00/3075. 
Vilanova y Piera (1863) realiza una descripción amplia de la provincia de Teruel desde varios puntos de vista. Entre ellos trata los materiales del Jurásico (1863: 46-72) y estudia las localidades turolenses donde estos afloran más continuamente. A modo de síntesis realiza un "Catálogo provisional de los principales fósiles jurásicos de la provincia de Teruel" (1863: 65), entre los que menciona 48 especies de bivalvos. En las láminas del Jurásico (láms. 9-10), figura únicamente especies de braquiópodos y de ammonites. Este autor hace especial mención al trabajo de Verneuil y Collomb (1852), indicando dentro de su Catálogo con un asterisco el material fósil que ha sido recogido por Verneuil. De las 48 especies de bivalvos citadas en este trabajo, 21 pertenecen al intervalo estratigráfico CallovienseKimmeridgiense y se indican en Apéndice.

De las demás especies mencionadas en intervalos estratigráficos precedentes, sólo la especie Pecten velatus Goldfuss, hoy atribuida al género Eopecten, y procedente de los materiales del Lías de Abejuela (provincia de Teruel), se ha encontrado en los materiales tratados en este trabajo.

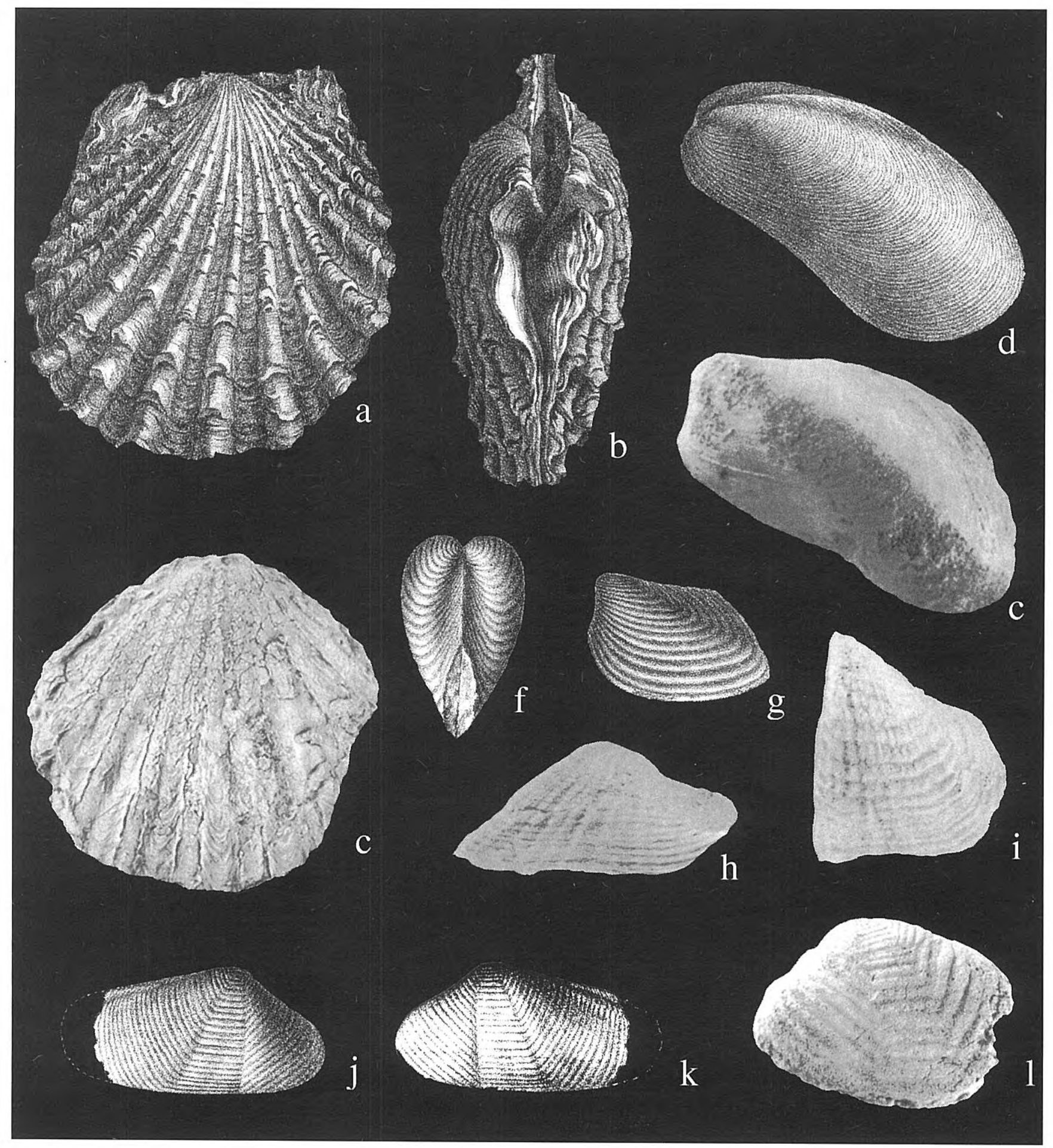


Donayre (1873) realiza un estudio geológico sobre la provincia de Zaragoza. Dedica un apartado al Sistema Jurásico (1873: 72-78) donde explica las rocas y fósiles existentes en las distintas localidades de la provincia. A modo de anexo, realiza un "Catálogo de los fósiles recogidos en la provincia de Zaragoza", donde indica el nombre de la especie, su edad y localidad donde se ha encontrado. De dicho catálogo, 8 especies son de bivalvos, y las mencionadas en el Apéndice se han encontrado posiblemente en edades equivalentes a las que este trabajo recoge.

Mallada (1879-1885) menciona más de 100 especies de bivalvos del Jurásico en España, de las que figura 31. Entre 1879 y 1884 publica la totalidad de las láminas donde muestra las especies de todos los grupos del Jurásico a las que hace referencia en el Boletín de la Comisión del Mapa Geológico de España (Rábano y Gutiérrez Marco, 1999). De las 59 láminas, 8 contienen figuras de bivalvos y se han publicado cronológicamente de esta manera: láminas 30, 30A-B (1881), lámina 30C (1883), láminas 31-32 (1879), lámina 33 (1883) y lámina 34 (1884).

En 1885, se publica el tomo II de la Sinopsis de las especies fósiles encontradas en España, que incluye una recopilación de los sistemas Triásico y Jurásico con la reimpresión de partes publicadas entre 1879 y 1884. Las láminas en las que figuran los bivalvos no sufren ninguna modificación. Por último, en 1892, en el Catálogo de las especies fósiles encontradas en España, Mallada enumera una gran parte de las especies mencionadas en los trabajos anteriores, añadiendo las localidades donde se han encontrado éstas, incluyendo las mencionadas en la recopilación de 1885 y otras localidades españolas no citadas hasta el momento. Además, menciona 39 especies más de bivalvos del Jurásico, no figurando ninguna de ellas. En este último trabajo corrige algunos errores de sus publicaciones anteriores, como la atribución equivocada de varias especies a algunos autores.

De las especies incluidas por Mallada $(1885,1892)$ en el Jurásico Inferior o Sistema Liásico y entre la "Oolita Inferior" y la "Gran Oolita" (Bajociense-Bathoniense), ocho se han encontrado registradas entre el Calloviense y el Kimmeridgiense de la Rama Aragonesa de la Cordillera Ibérica. Son las siguientes: Mactromya aequalis Agassiz; Pecten cingulatus Goldfuss; Pecten textorius Schlotheim; Hinnites velatus (Goldfuss); Ostrea gregarea Sowerby; Arca (Cucullaea) concinna Phillips; Lima duplicata Sowerby y Pleuromya alduini (Brongniart).

La especie Lima proboscidea Sowerby (Mallada, 1879, 1885: lám. 31, figs. 1-2), actualmente incluida en el género Ctenostreon, fue figurada por Mallada, pero no situada en ninguna edad ni localidad. Se ha reconocido como una forma relativamente abundante en el Calloviense del sector nororiental de la Rama Aragonesa de la Cordillera Ibérica (Fig. 1, a-c)

Dentro del intervalo estratigráfico tratado en este estudio (Calloviense-Kimmeridgiense), 49 especies son citadas por Mallada, y se recogen en el Apéndice final. La información proporcionada, en cuanto a las localidades y edad de las especies, incluye la información recogida en ambos trabajos de 1885 y 1892.

Cortázar (1885) recolecta más de 200 especies de fósiles en el Jurásico en la provincia de Teruel; muchas de ellas, como él mismo indica, ya habían sido recogidas por Verneuil, Collomb, Vilanova y Piera y otros geólogos de la época. Separa los materiales del Jurásico en los pertenecientes al "Liásico" y al "Grupo Oolítico" y va recorriendo la provincia de Teruel mencionando sin figurar las especies jurásicas encontradas en cada localidad, a veces haciendo referencia a otros autores. De las especies fósiles jurásicas citadas, 90 pertenecen a bivalvos, 46 de ellas correspondientes al intervalo Calloviense-Kimmeridgiense. De las especies liásicas que menciona, las siguientes se han encontrado en nuestro estudio: Ostrea gregarea Sowerby, Pecten velatus Goldfuss, Pecten textorius Schlotheim y Lima proboscidea Sowerby.

La lista de especies aportada por este autor incluye las especies recogidas por él y las mencionadas por otros autores previos. El autor señala con un asterisco aquéllas cuya existencia no ha comprobado en la provincia de Teruel. Las citadas en el "Grupo Oolítico" (Calloviense y Oxfordiense) se resumen en el Apéndice.

Palacios (1893) estudia la geología de la provincia de Zaragoza, separando los materiales en el "terreno Liásico" y en el "terreno Jurásico". Los datos paleontológicos indicados en este trabajo son escasos, y hacen referencia especialmente a los ammonites con los que data las series. De las especies fósiles mencionadas, 6 pertenecen a bivalvos del Liásico, de las que ninguna ha sido encontrada en este trabajo. En los materiales del "Jurásico" no menciona ninguna especie de bivalvos.

Dereims (1898) realiza un estudio estratigráfico detallado sobre el sur de Aragón. El periodo Jurásico lo divide en Inferior, Medio y Superior. Describe secciones de detalle más representativas y menciona detalladamente los autores previos y contemporáneos que estudiaron estos materiales. El autor cita, sin ilustrar, numerosas especies fósiles, especialmente de ammonoideos, con las que establece una sucesión bioestratigráfica correlacionable con la existente en otros países de Europa. Dentro de las formas de bivalvos, el autor reseña cuatro géneros y 49 especies; se observa un decrecimiento de los mismos desde el Jurásico Inferior al Superior. Entre las especies mencionadas en el Jurásico Inferior, las siguientes se han encontrado en el presente trabajo: Pleuromya alduini (Brongniart) y Pecten textorius Schlotheim. Las especies citadas en el Jurásico Superior son 14 y se recogen en el Apéndice.

\section{SIGLOS XX Y XXI}

Navás, en una nota geológica publicada en 1903, describe someramente una visita a la cueva de la Sima en Ricla (provincia de Zaragoza). Además de mencionar la fauna, flora y minerales que observa, cita nueve especies de bivalvos en el barranco de las conchas, todas ellas procedentes de materiales del Lías. 
Bataller (1954) enumera, sin figurar ninguna, las especies del Triásico y del Jurásico de España, separadas en grandes grupos. El autor indica la referencia de la diagnosis original, la edad y la localidad donde han sido encontradas. Respecto a los bivalvos del Jurásico, menciona ocho especies, todas ellas encontradas en el Jurásico Inferior (Liásico) de varias provincias españolas: Soria, Mallorca, Guadalajara, Lérida y Asturias.

Ninguna de las especies mencionadas por estos dos últimos autores ha sido identificada en este trabajo.

Castell et al. (1955) y Castell y de la Concha (1956) en su confección de la hoja de Maranchón estudian varios yacimientos fosilíferos liásicos. En sus trabajos ofrecen un listado de especies reconocidas de varios grupos taxonómicos, y la edad a la que las han atribuido. Además, describen y figuran algunas especies de bivalvos. De ellas, las especies Lopha gregarea Sowerby, Ctenostreon proboscideum Sowerby, Chlamys textoria Schlotheim, Gervillia aff. aviculoides Sowerby y Ceromya concentrica Sowerby han sido reconocidas en nuestro trabajo.

Westermann (1957) realiza un trabajo de tipo bioestratigráfico en los materiales del Jurásico de la Sierra de la Demanda. Los cuatro cortes geológicos que trata se sitúan en varias localidades de las provincias de Burgos y Soria. A pesar de que su estudio es básicamente bioestratigráfico, cita las especies identificadas de varios grupos fósiles. Entre los bivalvos mencionados, ninguna de las especies coincide con las reconocidas en nuestro trabajo. En los materiales del Jurásico Medio (Bajociense-Bathoniense) indica la presencia de pectínidos, límidos y pholadómidos, entre otros.

Almela y Sanz (1958) realizan una descripción sobre la historia geológica de España en la que dedican un apartado al Sistema Jurásico (1958: 95-102). Describen varios aspectos generales del Jurásico a escala mundial y se centran posteriormente en "las manchas jurásicas en España". En una tabla citan los fósiles característicos del Jurásico, sin indicar la procedencia de los mismos. De los fósiles que mencionan, ocho son especies de bivalvos (láms. 24-27, 31, 34), entre las que citan la especie Exogyra virgula d'Orbigny de los materiales del Kimmeridgiense (lám. 34, fig. 2). De las especies que pertenecen al Jurásico Inferior y Medio, Ctenostreon proboscideum (J. Sowerby) (lám. 26, fig. 2) se ha encontrado en este trabajo.

Riba (1959) en su Estudio geológico de la Sierra de Albarracín dedica un apartado al Lías y otro al Jurásico Medio y Superior (1959: 135-171). Hace una revisión exhaustiva de los autores anteriores a él que han estudiado la Sierra de Albarracín, y describe en detalle los perfiles estratigráficos. En cada uno de ellos menciona los fósiles encontrados, sin figurar ninguno. Los bivalvos que pertenecen al Jurásico Medio y Superior se han recogido en el Apéndice.

Bataller (1963) estudia algunos yacimientos de edad Calloviense situados en Abejuela (Teruel), en los que enumera las formas conocidas de ammonites, belemnites, braquiópodos, nautiloideos, gasterópodos y bivalvos. Estos dos últimos grupos los considera muy poco representados. Entre los bivalvos menciona Ostrea sp. (1963: 142). El único ejemplar de Ostrea sp. lo describe someramente (p. 171), comparándolo con Plicatula peregrina y Exogyra nana. En este mismo trabajo, el autor realiza una comparación entre el yacimiento de Abejuela y algunos yacimientos del Levante español. En el Puerto de Tortosa y en Cap de Salou, menciona la especie Posidonomya alpina A. Grass; y en la región de Vandellós, las especies Pholadomya reticulata Agassiz, Posidonomya alpina A. Grass y Trancredia cossmanni.

Behmel y Geyer (1966) realizan un estudio estratigráfico y paleontológico sobre el Jurásico Inferior de la Sierra de Albarracín. Entre el material figurado presentan siete especies de bivalvos (láms. 4-5). De éstas, dos se encuentran en el intervalo estudiado en este trabajo. El ejemplar figurado como Arcostrea sp. (lám. 4, fig. 4) podría asignarse a Actinostreon gregareum (J. Sowerby), y Velata tumida (Zieten) (lám. 5, fig. 3) podría asignarse a Eopecten velatus (Goldfuss). Estas determinaciones están de acuerdo con M. Gahr (com. personal 1999).

Goy (1971) trata los materiales del Jurásico Inferior (Pliensbachiense-Toarciense) al noreste de la provincia de Guadalajara. En esta región estudia tres cortes y cita los grupos fósiles registrados. Respecto a los bivalvos, de todas las especies reconocidas, ninguna se ha encontrado en nuestro trabajo.

Martel (1971) estudia dos series jurásicas situadas en las provincias de Cuenca y Valencia. Además de la descripción de los materiales del Lías hasta los del Portlandiense, incluye un listado de las especies reconocidas. Los grupos que predominan son los ammonites y los braquiópodos. Entre los bivalvos, cita algunos "pholadomyidos" en los materiales del Jurásico Inferior, y la especie Ostrea gregaria (Sowerby) en el Jurásico Superior.

Mouterde (1971) trata sobre los materiales del Lías en la localidad de Obón y sus alrededores. Cita muy pocas especies de bivalvos y ninguna de ellas coincide con las registradas en nuestro trabajo.

Meléndez Hevia y Ramírez del Pozo (1972) dedican su trabajo a los materiales del Jurásico de la Serranía de Cuenca. En cada uno de los tramos litológicos descritos incluyen una lista de especies de todos los grupos fósiles reconocidos, y figuran un gran número de ellas. Entre los bivalvos figurados, todos ellos del Jurásico Inferior, tan sólo la especie Lopha gregaria Sowerby está presente en nuestro trabajo.

Morillo-Velarde y Meléndez Hevia (1972) realizan un estudio de tipo estratigráfico y tectónico en el sector comprendido entre Soria y Burgos, centrando su máximo interés en los materiales del Jurásico. Entre los bivalvos mencionados en el Jurásico Inferior y Medio, sólo la especie Ctenostreon proboscideum Sowerby se ha reconocido en nuestro trabajo.

Viallard (1973: 135) cita en el sector de La Yesa (provincia de Valencia), dentro de los niveles del Kimmeridgiense, la presencia de grandes pteriidos, Modiolus y Myophorella (Jaworskiella) kobyi (Choffat). Indica la presencia de las especies Nucula inconstans 
Roeder y Astarte percrassa Étallon, en el Oxfordiense medio del sector de Talayuelas (provincia de Cuenca). También menciona (1973:146-147), en las margas y arcillas calcáreas del Kimmeridgiense inferior, en los Montes Universales (Hoya del Peral, Valencia), varias especies de bivalvos: Leda? roederi de Loriol, Nucula inconstans Roeder, Plicatula quenstedti de Loriol, Astarte cotejeani de Loriol, Astarte percrassa Étallon y Lucina berlieri de Loriol. De todas ellas, la primera, actualmente atribuida al género Dacryomya, se ha encontrado en Ricla, en los materiales de la Fm. Sot de Chera, de edad Oxfordiense superior-Kimmeridgiense. Dentro de los materiales aalenienses del sector Huérguina-Paravientos (provincia de Cuenca), menciona las especies citadas por Le Joncour (1965): Camptonectes cf. lens Sowerby y Chlamys dewalqui Oppel.

Sequeiros et al. (1978) dedican su trabajo al Jurásico Inferior y Medio del sector Belchite-Almonacid de la Cuba (provincia de Zaragoza). El estudio es básicamente bioestratigráfico y se ocupa de los ammonites, braquiópodos y bivalvos. En los materiales del Jurásico Inferior, citan varias especies de bivalvos, las siguientes se han reconocido en nuestro trabajo: Ceratomya concentrica (Sowerby), Lopha solitaria (Sowerby), Ctenostreon proboscidea (Sowerby) y Lopha gregaria (Sowerby). Del Jurásico Medio sólo citan la especie Posidonomya alpina (Gras.), que no ha sido reconocida en nuestro estudio.

Entre los estudios más recientes sobre bivalvos, es obligado citar dos trabajos que tratan el grupo de los bivalvos desde un punto de vista paleoecológico. Mayoral y Sequeiros (1979) realizan un estudio sobre los epizoos y "borers" en sustratos orgánicos entre los que se encuentran algunas especies de bivalvos (Tabla 3, p. 125), además de otros moluscos (gasterópodos, ammonites, nautiloideos y belemnoideos), crinoideos y braquiópodos, en el Jurásico Inferior y Medio de Belchite (provincia de Zaragoza). En un trabajo posterior, los mismos autores (Sequeiros y Mayoral, 1980) realizan un estudio semejante sobre la colonización por epizoos de conchas de la especie Plagiostoma gigantea (Sowerby), procedentes del Toarciense de Belchite (provincia de Zaragoza).

Goy y Suárez Vega (1983) realizan un trabajo de síntesis en el que describen el Jurásico de España desde los puntos de vista estratigráfico y paleogeográfico, a la vez que mencionan los principales grupos de fósiles encontrados, especialmente ammonoideos. Señalan la existencia de algunos niveles más relevantes caracterizados por la presencia de bivalvos dentro de los materiales de la parte superior de la Fm. Cortes de Tajuña (algunos ejemplares de Gryphaea). Limitando la parte superior de la Fm. Cuevas Labradas observan una o más superficies ferruginizadas con ostreidos. Por último, en el área de Moscardón, en el Bathoniense superior, citan la presencia de valvas desarticuladas de la especie Bositra buchii.

Meléndez et al. (1983) realizan un estudio de tipo bioestratigráfico sobre el Oxfordiense superior en Moscardón (Teruel), en el que tratan detalladamente el grupo de los ammonoideos y dan una visión somera de otros grupos de moluscos (belemnites, gasterópodos y bivalvos). Respecto al último grupo, que es el que nos atañe, mencionan formas de pequeño tamaño cercanas a Nicaniella (Trautscholdia). Por la descripción realizada (1983: 39), parece bastante claro que se trata de la especie Nicaniella (Trautscholdia) carinata (Phillips), cuyo registro es muy abundante en la Fm. Sot de Chera (Oxfordiense superior-Kimmeridgiense inferior) de la Rama Aragonesa de la Cordillera Ibérica. Las valvas más aplanadas que describen a continuación y que relacionan con la especie Astarte prismatica Étallon, podrían pertenecer a los géneros Astarte o Nicaniella (Nicaniella). También mencionan, con ciertas dudas, la presencia de algunos moldes atribuibles al género Placunopsis (1983: 39, 42).

En los últimos años los bivalvos del Jurásico Inferior han sido objeto de diversos estudios. Bernad y Calvo (1995) y Bernad (1999b) realizan un estudio taxonómico y estadístico de la especie Plicatula spinosa Sowerby, procedente del Toarciense inferior de Turmiel (provincia de Guadalajara), concluyendo que esta especie se comporta como oportunista en un ambiente de baja energía.

Bernad (1996a,b) se ocupa del apartado de los bivalvos en la excursión realizada en la Cordillera Ibérica durante el $1^{\text {st }}$ Toarcian and $4^{\text {th }}$ Aalenian working groups Meeting. En el tránsito Pliensbachiense-Toarciense del sector La Almunia de Doña Godina y Ricla (provincia de Zaragoza), (1996a: 14, figs. 1.5-1.6), describe dos tramos bien diferenciados. El primero de ellos abarca desde la parte superior del Pliensbachiense hasta la Subzona Mirabile (Biozona Tenuicostatum) del Toarciense inferior, donde la abundancia y la diversidad de bivalvos (Pholadomyoida, Pteroida y Veneroida) es relativamente alta. El segundo tramo incluye el resto de las biozonas del Toarciense, en donde la abundancia y diversidad de bivalvos se reducen notablemente, estando presentes únicamente escasos restos de la Superfamilia Pectinacea. De las especies citadas por el autor, las siguientes se han encontrado en la Rama Aragonesa de la Cordillera Ibérica, en el intervalo Calloviense-Kimmeridgiense: Pseudolimea duplicata (Sowerby), Chlamys textoria (Schlotheim) y Pleuromya alduini (Brongniart). El Toarciense de la Rambla del Salto (Sierra Palomera, provincia de Teruel) se caracteriza por el notable incremento de la abundancia y diversidad de los bivalvos en todas las biozonas (1996b: 36, figs. 2.6-2.8). Siete órdenes están representados: Pteroida, Arcoida, Mytiloida, Trigonioida, Nuculoida, Veneroida y Pholadomyoida. Dentro de ellos, las siguientes especies se han encontrado en el intervalo estudiado: Chlamys textoria (Schlotheim), Pseudolimea duplicata (Sowerby), Pleuromya alduini (Brongniart), Pleuromya uniformis (Sowerby) y Lopha [=Actinostreon] gregarea (Sowerby). Por último, en el tránsito Toarciense-Aaleniense del área de Fuentelsaz (provincia de Guadalajara), Bernad (1996c: 62, fig. 3.5) indica que el máximo de abundancia y diversidad se registra en la Subzona Mactra y que decrece en la Subzona Aalensis para cambiar esta tendencia de nuevo durante la Subzona Buckmani (todas ellas correspondientes al Toarciense superior, Biozona 
Aalensis). Durante la Biozona Opalinum (Aaleniense) la diversidad y abundancia de los grupos de bivalvos decrece sensiblemente, encontrándose representados únicamente los órdenes Pteroida, Trigonioida, Arcoida, Mytiloida, Veneroida y Pholadomyoida. De todas las especies citadas por el autor, sólo Pseudolimea duplicata (Sowerby) se encuentra en este trabajo.

Bernad (1997a) da a conocer las especies de bivalvos del Lías español depositadas en el Museo Geominero (IGME) de Madrid. La colección alojada en este Museo consta de 462 ejemplares; el autor actualiza las denominaciones taxonómicas preexistentes indicando las localidades españolas donde se han encontrado dichos taxones. Además, figura 32 de las especies mencionadas. Las especies de bivalvos de esta colección que han sido encontradas en este trabajo son las siguientes: Pleuromya alduini (Brongniart), Modiolus bipartitus Sowerby, Gryphaea bilobata Sowerby, Pseudolimea duplicata (Sowerby), Eopecten velatus (Goldfuss), Chlamys textoria (Schlotheim) y Lopha gregarea (Sowerby).

Bernad (1997b) realiza una revisión de las especies atribuidas al género Plagiostoma correspondientes al Toarciense de la Cordillera Ibérica, de las cuales ninguna se encuentra en este trabajo.

Bernad (1999a) realiza una revisión de las especies de bivalvos figuradas por Torrubia (1754), que proceden de los alrededores de Molina de Aragón, de modo que determina dicho material, reproduciendo las figuras originales de Torrubia, y como comparación añade algunas figuras del material recogido por él mismo en algunas localidades de las provincias de Guadalajara y Teruel. De las especies mencionadas, Lopha [= Actinostreon] gregarea (J. Sowerby) se encuentra en el presente trabajo.

Los bivalvos del Jurásico Medio y Superior han sido muy someramente tratados por autores previos, tal como ha podido verse a lo largo de este capítulo, debido a la escasez de material en estos niveles y a su deficiente conservación. Los bivalvos del intervalo estratigráfico Calloviense-Kimmeridgiense han sido estudiados por Delvene en los últimos cuatro años. Entre los trabajos más relacionados con la taxonomía y paleoecología de bivalvos se destacan los siguientes. Delvene (1997a), en la Tesis de Licenciatura, realiza un estudio de carácter taxonómico en el que identifica bivalvos pertenecientes a los órdenes Pholadomyoida, Veneroida, Mytiloida y Pteroida (subórdenes Pteriina y Ostreina), en el intervalo Calloviense-Oxfordiense del sector nororiental de la Cordillera Ibérica (provincias de Zaragoza y Teruel). Asimismo, se agrupan todos los taxones según los diferentes modos de vida indicando las proporciones de dichos grupos en los niveles más fosilíferos.

Los bivalvos son un grupo especialmente abundante en la Fm. Sot de Chera del área de Ricla (provincia de Zaragoza). En Delvene (1997b) se citan tres de las especies más frecuentes en estos materiales: Nicaniella (Trautscholdia) carinata (Phillips), Gervillella aviculoides (J. Sowerby) y Nanogyra nana (J. Sowerby). Los datos sobre la taxonomía de bivalvos, bioestratigrafía y algunos aspectos paleoecológicos de los materiales de la Fm. Sot de Chera en el sector de Ricla se concretan en Delvene et al. (1998).

Delvene (1998) realiza una revisión del trabajo presentado en 1997a, actualizando el nombre de algunos taxones, incorporando nuevo material y añadiendo algunos datos interpretativos de los hábitos de vida de los bivalvos en ese sector.

Por último, Delvene (1999) cita las especies bivalvos más abundantes durante el Calloviense y Oxfordiense en el sector más oriental de la Rama Aragonesa de la Cordillera Ibérica.

\section{CONCLUSIONES}

Los bivalvos del Jurásico Medio y Superior han sido someramente tratados hasta el momento, debido en parte a su escaso registro en comparación con otros grupos de moluscos, especialmente los ammonoideos, y también porque su conservación es bastante deficiente. Algunos autores figuran especies de bivalvos en materiales del Jurásico Inferior que también se han registrado en los materiales del Jurásico Medio y Superior, como se ha citado en páginas anteriores, pero los únicos autores que figuran especies de bivalvos encontradas en el Jurásico Medio y Superior son Verneuil y Collomb y Mallada (Fig. 1). Como conclusión de este trabajo se muestran las especies de bivalvos figuradas por estos autores en este intervalo de tiempo en comparación con las recientemente estudiadas (Delvene, 2001) pertenecientes a las formaciones Chelva, Yátova y Loriguilla de edad Calloviense-Kimmerdgiense de la Rama Aragonesa de la Cordillera Ibérica (Fig. 1).

\section{AGRADECIMIENTOS}

Este trabajo forma parte de la Tesis Doctoral realizada en Cotutela Europea entre la Universidad de Zaragoza (Dr. G. Meléndez) y la Universidad de Würzburg, Alemania (Prof. F. T. Fürsich). La Tesis se realizó gracias a una beca predoctoral del Gobierno de Aragón (CONSI+D) y varias ayudas para estancias de investigación en Alemania (Programa Europa, CAI, CONSI+D) y en España (Instituto de Estudios Turolenses, Museo Mas de las Matas). Es una contribución a los proyectos RF P35/97 (Gobierno de Aragón) y RF 2000/448 (Fundación Conjunto Paleontológico de Teruel). La autora agradece a la Dra. Z. Herrera (Univ. Zaragoza) la realización de las fotografías y a I. Pérez-Urresti (Univ. Zaragoza) la lectura crítica del manuscrito y su inestimable ayuda a lo largo de todo el trabajo realizado. Por último, quiero agradecer las sugerencias y comentarios recibidos, a los revisores de este trabajo (doctores Sixto Fernández López y Antonio Goy) y a la editora de la revista (Dra. María Luisa Martínez Chacón).

\section{BIBLIOGRAFÍA}

Almela, A. y Sanz, R. 1958. Resumen de la historia geológica de la Tierra. Memorias del Instituto Geológico y Minero de España, 59, 1-174. 
Bataller, J. R. 1954. Enumeración de las especies nuevas del Triásico y Jurásico de España. Estudios Geológicos, 10 (21), 49-77.

Bataller, J. R. 1963. El Caloviense de Abejuela (Teruel). Notas y Comunicaciones del Instituto Geológico y Minero de España, 69, 131-174.

Behmel, H. und Geyer, O. F. 1966. Beiträge zur Stratigraphie und Paläontologie des Juras von Ostspanien. III. Stratigraphie und Fossilführung im Unterjura von Albarracín (Provinz Teruel). Neues Jahrbuch für Geologie und Paläontologie. Abhandlungen, 124 (1), 1-52.

Bernad, J. 1996a. In: Goy, A., Martínez, G., Arias, C., Bernad, J., García Joral, F., Gómez, J. J., Herrero, C., Perilli, N. and Ureta, S. The Toarcian in the sector located between La Almunia de Doña Godina and Ricla. Fieldtrip Iberian Range Guide-book, $1^{\text {st }}$ Toarcian and $4^{\text {th }}$ Aalenian working groups Meeting, 3-23.

Bernad, J. 1996b. In: Comas-Rengifo, M.J., Gómez, J.J., Goy, A., Arias, C., Bernad, J., García Joral, F., Herrero, C., Martínez, G. and Perilli, N. The Toarcian in the Rambla del Salto (Sierra Palomera) section. Fieldtrip Iberian Range Guide-book, $1^{\text {st }}$ Toarcian and $4^{\text {th }}$ Aalenian working groups Meeting, 27-48.

Bernad, J. 1996c. In: Goy, A., Ureta, S., Arias, C., Bernad, J., Barrón, E., Canales, M.L., García Joral, F., Gialanella, P., Gómez, J.J., Herrero, C., Martínez, G., Osete, M.L., Perilli, N. and Villalaín, J.J. The Toarcian-Aalenian transition in Fuentelsaz section. Fieldtrip Iberian Range Guide-book, $1^{\text {st }}$ Toarcian and $4^{\text {th }}$ Aalenian working groups Meeting, 51-77.

Bernad, J. 1997a. Catálogo de los bivalvos del Lías español depositados en el Museo Geominero (ITGE; Madrid). Boletín Geológico y Minero, 108, 3-28.

Bernad, J. 1997b. El género Plagiostoma en el Toarciense de la Cordillera Ibérica. Comunicaciones del IV Congreso del Jurásico de España, 45-46.

Bernad, J. 1999a. In: Goy, A. y Rodrigo, A. Tras las huellas de Torrubia (1698-1761) por el Señorío de Molina. Guía de la Excursión. XV Jornadas de Paleontología, 33 pp.

Bernad, J. 1999b. Plicatula spinosa Sowerby, en el Toarciense inferior (Jurásico) de Turmiel (Guadalajara, España). Boletín Geológico y Minero, 110, 227-236.

Bernad, J. 1999c. In: Goy, A., Ureta, S., Arias, C., Barrón, E., Bernad, J., Canales, M.L., García Joral, F., Gialanella, P. R., Gómez, J.J., Herrero, C., Martínez, G., Osete, M.L., Perilli, N. and Villalain, J.J. The Fuentelsaz Section (Guadalajara, Iberian Range, Spain): A possible global geosite. Towards The Balanced Management and Conservation of the Geological Heritage in the New Millenium (Eds. D. Barettino, M. Vallejo and E. Gallego), Madrid, 228-233.

Bernad, J. y Calvo, A. 1995. Plicatula spinosa (Sowerby) en el Toarciense inferior de Turmiel (Guadalajara). XI Jornadas de Paleontología, 51-53.

Castell, J. y de la Concha, S. 1956. Mapa Geológico de España, escala 1:50.000. Explicación de la Hoja $n^{\circ} 462$, Maranchón (Guadalajara). Instituto Geológico y Minero de España, 42 pp.

Castell, J., de la Concha, S. y de la Revilla, J. 1955. Un yacimiento fosilífero del Lías de Maranchón (Guadalajara). Notas y Comunicaciones del Instituto Geológico y Minero, 40, 25-40.

Cortázar, D. 1885. Bosquejo físico, geológico y minero de la provincia de Teruel. Boletín de la Comisión del Mapa Geológico de España, 12, 345 pp.

Delvene, G. 1997a. Estudio taxonómico e implicaciones paleoecológicas de las asociaciones de Bivalvos del Calloviense y Oxfordiense Medio y Superior en la Cordillera Ibérica Nororiental. Tesis de Licenciatura, Universidad de Zaragoza, 144 pp. (inédita).

Delvene, G. 1997b. Estudio paleontológico de los Bivalvos de la Formación Sot de Chera en Ricla, Zaragoza (Oxfordiense Superior, Cordillera Ibérica). Comunicaciones del IV Congreso de Jurásico de España, 61-62.

Delvene, G. 1998. Bivalvos del Jurásico Medio y Superior de la Cordillera Ibérica Nororiental (Ariño y Oliete, España). Coloquios de Paleontología, 49, 55-71.

Delvene, G. 1999. Jurassic Bivalves of the Iberian Range (Spain). Biology and Evolution of the Bivalvia. Malacological Society of London, 58.

Delvene, G. 2001. Middle and Upper Jurassic bivalves from the Iberian Range (Spain). Beringeria, 28, 43-104.

Delvene, G., Ramajo, J. y Pérez-Urresti, I. 1998. Nuevos datos sobre Paleontología y Bioestratigrafía en la Formación Sot de Chera (Jurásico Superior) de Ricla, España. Coloquios de Paleontología, 49, 73-91.

Dereims, A. 1898. Reserches géologiques dans le Sud de l'Aragon, 199 pp.

Donayre, F. M. 1873. Bosquejo de una descripción física y geológica de la provincia de Zaragoza. Comisión del Mapa Geológico de España, 128 pp.

Ezquerra, J. 1850-1857. Ensayo de una descripción general de la estructura geológica del terreno de España en la Península. Memorias de la Real Academia de Ciencias de Madrid 1 (parte $1^{\mathrm{a}}$ ): $35-65$ [1850]; 1 (parte $3^{\mathrm{a}}$ ): 161 184 [1854]; 4 (parte 2a): 351-394 [1857].

Goy, A. 1971. El límite Pliensbachiense-Toarciense en Establés (Guadalajara). Estudios Geológicos, 27, 297. 304.

Goy, A. y Suárez Vega, L. C. 1983. El Jurásico. In: Geología de España. Libro Jubilar J.M. Ríos, 2, I.G.M.E., 63-79.

Le Joncour, M. 1965. Description géologique des environs de Cañete (province de Cuenca, Espagne). Diplome des Études Spécialisés, Univ. de Dijon, 83 pp. (inédito).

Mallada, L. 1879. Sinopsis paleontológica de España. Láminas. Boletín de la Comisión del Mapa Geológico de España, 6, láms. 21, 23, 31-32, 34, 36, 41-42 (Jurásico).

Mallada, L. 1881. Sinopsis paleontológica de España. Láminas. Boletín de la Comisión del Mapa Geológico de España, 8, láms. 1-2, 8-10, 14, 18-19, 24-26, 30, 30A-B, 38, 38A (Jurásico).

Mallada, L. 1883. Sinopsis paleontológica de España. Láminas. Boletín de la Comisión del Mapa Geológico de España, 10, lám. 30C, 33 (Jurásico).

Mallada, L. 1884. Sinopsis paleontológica de España. Láminas. Boletín de la Comisión del Mapa Geológico de España, 11, láms. 12, 27, 43 (Jurásico). 
Mallada, L. 1885. Sinopsis de las especies fósiles que se han encontrado en España. Terreno Mesozoico (Sistemas Triásico y Jurásico), 2, 16 pp., láms. 1-3 (Triásico); iiixii, 150 pp., láms. 1-28, 28A-F, 29, 29A-B, 30, 30A-C, 31-34 (Jurásico).

Mallada, L. 1892. Catálogo general de las especies fósiles encontradas en España. Boletín de la Comisión del Mapa Geológico de España, 18, 253 pp.

Martel, M. 1971. El Jurásico de la región Contreras-Sierra de la Bicuerca. Cuadernos de Geología Ibérica, 2, 401416.

Mayoral, E. y Sequeiros, L. 1979. Significado paleoecológico de algunos epizoos "borers" del Jurásico Inferior y Medio de Belchite (Zaragoza, Cordillera Ibérica). Cuadernos de Geología Ibérica, 10, 121-135.

Meléndez, G., Olóriz, F. y Sáez, A. 1983. Nuevos datos bioestratigráficos sobre el Oxfordiense superior en Moscardón (Teruel). In: Libro Jubilar J.M. Ríos. Contribuciones sobre temas generales, 3, I.G.M.E., 3344.

Meléndez Hevia, F. y Ramírez del Pozo, J. 1972. El Jurásico de la Serranía de Cuenca. Boletín Geológico y Minero, 83-84, 313-342.

Morillo-Velarde, M. J. y Meléndez Hevia, F. 1972. "La falla de San Leonardo": interpretación paleogeográfica (Cordillera Ibérica, Soria-Burgos). Estudios Geológicos, 28, 65-76.

Mouterde, R. 1971. Une coupe du Lias a Obon aux confins des provinces de Teruel et de Saragosse. Cuadernos de Geología Ibérica, 2, 345-354.

Navás, L. 1903. La cueva de la Sima en Ricla (Zaragoza). Boletín de la Sociedad española de Historia Natural, 3, 62-65.

Palacios, P. 1893. Reseña geológica de la región meridional de la provincia de Zaragoza. Boletín de la Comisión del Mapa Geológico, 19, 1-112.

Pelayo, F. 1994. El Aparato para la Historia Natural Española de José Torrubia (1698-1761): diluvismo, gigantes y la naturaleza de los fósiles, en el pensamiento español del siglo XVIII. In: Edición facsímil del Aparato para la Historia Natural Española, 3-45.

Rábano, I. y Gutiérrez-Marco, J. C. 1999. La "Sinopsis" paleontológica de Lucas Mallada: fechas de publicación y otros aspectos editoriales. Temas Geológico-Mineros ITGE, 26, 103-110.

Riba, O. 1959. Estudio geológico de la Sierra de Albarracín. Instituto "Lucas de Mallada" de investigaciones geológicas. Sección de Geomorfología de Barcelona. Instituto de Estudios Turolenses. Monografías, 16, 283 pp.

Sequeiros, L. y Mayoral, E. 1980. Epizoos y perforantes sobre Plagiostoma gigantea (Sow.), Bivalvia (Jurásico Inferior); un modelo de relaciones entre paleoecología y diagénesis. Revista del Instituto de Investigaciones Geológicas, 34, 149-159.

Sequeiros, L., Cólera, I., Valenzuela, R. y Sánchez, I. 1978. Bioestratigrafía del Jurásico (Lías y Dogger) en el sector Belchite-Almonacid de la Cuba (provincia de Zaragoza, Cordillera Ibérica). Estudios Geológicos, 34, 293-298.

Torrubia, J. 1754. Aparato para la Historia Natural Española, 1, 204 pp.

Torrubia, J. 1754. Aparato para la Historia Natural Española. In: Reproducción facsímil del tomo I de la obra, edición de 1754, y de parte de su traducción al alemán, edición de 1773. UEI de Paleontología e Instituto de Geología Económica (eds.), 375 pp.

Viallard, P. 1973. Recherches sur le cycle Alpin dans la Chaîne Ibérique Sud-Occidentale. Tesis Doctoral, Universidad Paul Sabatier-Toulouse, 445 pp. (inédita).

Verneuil, E. de et Collomb, E. 1852. Coup d'oeil sur la constitution géologique de quelques provinces de l'Espagne. Bulletin de la Societé Géologique de France, $2^{a}$ série, 61-147; 162-167.

Vilanova y Piera, J. 1863. Ensayo de descripción geognóstica de la provincia de Teruel, en sus relaciones con la agricultura de la misma, 312 pp.

Westermann, G. 1957. Investigaciones bioestratigráficas en el Jurásico, al sur de la Sierra de la Demanda (N. de España). Notas y Comunicaciones del Instituto Geológico y Minero de España, 45, 181-214.
Manuscrito recibido: 27 de julio, 2001 Manuscrito aceptado: 31 de enero, 2002

\section{APÉNDICE}

\section{Bivalvos del Calloviense-Kimmeridgiense discutidos por} Verneuil y Collomb (1852):

Mytilus bipartitus Goldfuss (Frías; "Kelloway")

Pecten subspinosus Schlotheim (entre Alustante y Prados Redondos; "Oxfordien")

Pecten textorius Goldfuss (Anchuela; "Lias Supérieur")

Pholadomya trapezina Buvignier (entre Frías y Villar del Cobo; "Oxfordien"; en España, en el Oxfordiense)

Ostrea gregarea Sowerby (Guadalaviar, Griegos, Anchuela, Albarracín, Villar del Cobo, Checa, Carrascosa, Torremocha, Barahona; "Kelloway y Oxfordien"; en España, en el Lías)
Bivalvos del Jurásico citados por Ezquerra (1854). -Especies identificadas en el Calloviense-Kimmeridgiense de la Rama Aragonesa de la Cordillera Ibérica:

Astarte elegans Sowerby (Torres)

Gryphaea incurva Sowerby (Albarracín, Calomarde)

Lima elea d'Orbigny (Brieva, Anchuela)

Lima gigantea Deshayes (Ablanque, Anchuela)

Lima obscura Deshayes (Albarracín)

- Lima proboscidea Sowerby (Ablanque, Alcolea del Pinar, Canales (prov. de Logroño), Mansilla de la Sierra)

Lithodomus prolongus d'Orbigny (Calomarde)

Lutraria rotundata Goldfuss (Anchuela, Monterde) 
Lyonsia unioides Goldfuss (Anchuela, Villar del Cobo) Mactromya liasina Agassiz (Maranchón, Albarracín, Villar del Cobo)

- Mytilus bipartitus Goldfuss (Frías)

Mytilus plicatus ? Sowerby (Las Majadas, Albarracín)

Opis sarthacensis d'Orbigny (Anchuela)

Ostrea erina d'Orbigny (Anchuela)

Bivalvos del Calloviense-Kimmeridgiense mencionados por Vilanova y Piera (1863). *Material fósil recogido por Verneuil **Material fósil recogido por Verneuil y Vilanova y Piera. El material sin asterisco pertenece a Vilanova y Piera. •Especies identificadas en la Rama Aragonesa de la Cordillera Ibérica:

- Arca concinna Goldfuss (Torrevelilla; "Oxfórdico, Portlándico")

- Ceromya excentrica Agassiz (entre Josa y Obón; "Kimmeridgico")

Ceromya inflata Agassiz (Torrevelilla y Obón; "Oxfórdico, Kimmeridgico, Portlándico")

Lima pectiniformis Brongniart (Obón, Ariño; "Grande Oolita")

-Lima proboscidea Sowerby (Obón, Ariño; "Grande Oolita")

Lima substriata ? Münster (Obón, Ariño; "Grande Oolita")

Modiola acinaces Leymerie (Ariño; "Coralrag")

Mya rugosa Roemer (Torrevelilla, Torres; "Portlándico")

-**Mytilus bipartitus Goldfuss (Albarracín, Frías; "Oxfórdico")

Mytilus pectinatus Sowerby (Albarracín; "Oxfórdico")

Mytilus sublaevis Sowerby (Albarracín; "Oxfórdico")

* Ostrea colubrina Goldfuss (Guadalaviar, Obón, Griegos, Albarracín, Villar; "Calóvico")

•**Ostrea gregarea Sowerby (Guadalaviar, Obón, Griegos, Albarracín, Villar, etc.; "Oxfórdico")

Pecten barbatus ? Sowerby (Obón; "Oxfórdico")

Pecten lens Sowerby (Albarracín; "Oolita inferior y Oxfórdico")

Pecten lugdunensis Sowerby (Torrevelilla; "Oolita inferior y Portlándico")

**Pholadomya paucicosta Roemer (Albarracín; "Coralrag")

-*Pholadomya trapezina Buvignier (entre Frías y Villar; "Oxfórdico")

Pleuromya helena Chapuis \& Dewalque (Torres; "Grande Oolita")

*Thracia chauviniana d'Orbigny (Frías; "Oxfórdico")

*Trigonia clathrata ? Agassiz (Albarracín; "Oxfórdico")

Bivalvos del Jurásico citados por Donayre (1873). -Especies identificadas en la Rama Aragonesa de la Cordillera Ibérica:

- Ceromya excentrica d'Orbigny (Aguilón; Jurásico, “grupo oolítico o Lías Superior")

Lima punctata Deshayes (Aguilón; Jurásico, "grupo oolítico o Lías Superior")

Pecten heblii d'Orbigny (Aguilón; Jurásico, "grupo oolítico o Lías Superior")

-Pholadomya trapecina? Buvignier (Aguilón; Jurásico, "grupo oolítico o Lías Superior")
Bivalvos del Calloviense-Kimmeridgiense mencionados por Mallada $(1885,1892)$. Especies identificadas en la Rama Aragonesa de la Cordillera Ibérica:

Anisocardia tyrolensis, Zitt. (Fuente de los Frailes de Cabra; Titónico)

Arca (Cucullaea) texta Roemer (Río Arcos; Kimmeridgiense-Portlandés)

Astarte bulla (Peña Escorxada de Castelldefels; Kimmeridgiense)

Aucella carinata Paronna sp. (Fuente de los Frailes de Cabra; Titónico)

Aucella zitteli, Neum. (Segura de la Sierra; Kimmeridgiense) ?Cardium dissimile Sowerby (entre Chinchilla y Almansa?; Calloviense Kimmeridgiense)

- Ceromya excentrica Voltz. sp. (entre Josa y Obón, Río Arcos, Anchuela, Aguilón?, El Pobo, Alcublas, Jérica, entre Chinchilla y Almansa; niveles inferiores del Jurásico, Oxfordiense)

Ceromya inflata Voltz. sp. (Obón, Torrevelilla, Albarracín, entre Anguela y Hombrados, entre Chinchilla y Almansa; Oxfordiense-Portlandés)

Corbula pichleri Zitt. (Fuente de los Frailes de Cabra?; Kimmeridgiense)

Eligmus polytypus Desl. (El Chorro; Kimmeridgiense)

- Goniomya trapezina (Buvignier) (entre Frías y Villar del Cobo; Oxfordiense)

Hinnites paniscus d'Orbigny (Albarracín; ?Jurásico Medio y Superior)

Hinnites tenuistriatus d’Orbigny (Sarrión; ?Jurásico Medio y Superior)

Lima rigida Sowerby (entre Clarés y Mochales; Oxfordiense)

Lucina rugosa Roemer sp. (Torres?, Torrevelilla?, Alcublas?; Kimmeridgiense)

Lyonsia sulcosa Agassiz (Ligüerzana; Oxfordiense)

Mactromya rugosa Roemer sp. (Torres?, Torrevelilla?; Portlandés y Kimmeridgiense)

Mytilus acinaces Leym. sp. (Ariño?; "Coralino")

-Mytilus bipartitus Sowerby (Albarracín, Frías, Griegos; Oxfordiense)

Mytilus pectinatus Sowerby (Albarracín; Oxfordiense)

Mytilus sowerbyanus d'Orbigny (Albarracín, Javalambre, Majadas, La Cierva, Tragacete, Ricla, Calomarde, Guadalaviar, Tramacastilla, Anchuela del Campo; Bathoniense, Oxfordiense, "Gran Oolita")

Mytilus subpectinatus Sowerby (Albarracín, Guadalaviar, Torremocha; Oxfordiense)

Neoera lorioli Nem. (Cabra; Titónico)

Neoera mosensis Buvignier (entre Salsadella y Sierra de Valdanche; Kimmeridgiense, Portlandés)

Ostrea amata, d'Orbigny (Frías, Calomarde; Oxfordiense)

Ostrea bruntrutana Thurmann (entre Salsadella y Sierra de Valdanche, entre Castell de Cabres y Morella; Jurásico Superior, Oxfordiense)

Ostrea colubrina Lamarck (Villar del Cobo, Albarracín; "Coralino")

Ostrea deltoidea Lamarck (Reocín?, Udías?, Comillas?, Torcal de Antequera; Oxfordiense, "Coralino")

-Ostrea solitaria Sowerby (Ablanque; "Coralino")

Ostrea virgula Defrance (entre Salsadella y Sierra de 
Valdanche, Jérica?, Torcal de Antequera; "Coralino", Kimmeridgiense, Jurásico Superior)

?Pecten inaequicostatus Phillips (Albarracín?; Oxfordiense) Pecten lens Sowerby (Albarracín, entre Frías y Calomarde; Bajociense, Caloviense, Oxfordiense)

Pecten lugdunensis Sowerby (Torrevelilla?; Oxfordiense) Pecten sub-fibrosus d'Orbigny (Venta de los Baños, Ventas de Buñol; Oxfordiense)

-Pecten sub-spinosus Schlotheim (Alustante, Prados Redondos; Caloviense, Oxfordiense)

Pholadomya (Homomya) hortulana Agassiz (entre Chinchilla y Almansa; Oxfordiense)

- Pholadomya acuminata Hartmann (Frías, Albarracín; Oxfordiense)

Pholadomya escheri Agassiz (Foz de Calanda; Caloviense) Pholadomya exaltata Agassiz (Becerril?; Calloviense, Oxfordiense, Kimmeridgiense)

Pholadomya lineata Goldfuss (entre Campalbo y Manzanaruelo; Oxfordiense)

Pholadomya murchisoni Sowerby (Torremocha de los Arrieros, Pradilla, Montorio, Caramillo de la Fuente, Mochales, Anguela, Torremochuela?, Albarracín, Paracense, Sarrión, entre Josa y Hoz de la Vieja; "Oolita inferiorCaloviense")

Pholadomya paucicosta Roemer (Albarracín, Río Arcos, Ablanque; Oxfordiense)

Pholadomya pectinata Agassiz (Alcublas; Portlandés y Kimmeridgiense)

- Pholadomya protei Defrance (Río Arcos; Oxfordiense)

Pinna ampla Sowerby (Villel de Mesa; "Gran Oolita" y Oxfordiense inferior)

Posidonomya alpina Gras. (Las Hoyas de Loja; Kimmeridgiense)

Thracia chauviniana d'Orbigny (Frías; Oxfordiense)

Trigonia clavellata Sowerby (Lastres?; Oxfordiense)

Trigonia gibbosa Sowerby (Alcublas, El Toro; Oxfordiense)

Bivalvos del Calloviense-Oxfordiense citados por Cortázar (1885). - Especies identificadas en la Rama Aragonesa de la Cordillera Ibérica. Cortázar (1885) indica la no comprobación de la existencia de la especie en la provincia de Teruel, aunque la incluye por ser citada por autores anteriores:

- Astarte burgomontana Verneuil (Teruel?; "Grupo Oolítico")

Lima pectiniformis Brongniart (Teruel?; "Grupo Oolítico")

$\checkmark$ Lima substriata Münster (Teruel?; "Grupo Oolítico")

- Mactromya aequalis Agassiz (Royuela; "Grupo Oolítico")

$\diamond$ Modiola acinaces Leym. (Teruel?; "Grupo Oolítico")

$\checkmark$ Mya rugosa Roem. (Teruel?; "Grupo Oolítico")

$\checkmark$ Mytilus sublevis Sowerby (Teruel?; "Grupo Oolítico")

$\checkmark$ Pecten lugdunensis Sowerby (Teruel?; "Grupo Oolítico")

$\diamond$ Pholadomya bucardium Agassiz (Teruel?; "Grupo Oolítico")

$\diamond$ Pleuromya helesia Chapuis et Dur. (Teruel?; "Grupo Oolítico")

$\diamond$ Pholadomya paucicosta Roem. (Teruel?; "Grupo Oolítico")

- $\diamond$ Pholadomya trapezina Buvignier (Teruel?; "Grupo
Oolítico")

Thracia chauviniana d’Orbigny (Teruel?; "Grupo Oolítico")

$\checkmark$ Trigonia clathrata Aggasiz (Teruel?; "Grupo Oolítico")

$\checkmark$ Trigonia costata Park. (Teruel?; "Grupo Oolítico")

- Arca concinna? Phillips (Valdecomadres, Virgen del Carmen, Bco. Hondo, Bco. Argudo, Pajares, Valdemarín, Entrambasaguas, Albarracín, Cuesta de la Cerroja, Cuesta del Pozuelo, Guadalaviar; "Grupo Oolítico")

Arca texta d'Orbigny (Río Arcos; "Grupo Oolítico")

- Ceromya excentrica Agassiz (Pobo, Río Arcos; "Grupo Oolítico")

Ceromya inflata Voltz. (Valdecomadres, Virgen del Carmen, Bco. Hondo, Bco. Argudo, Pajares, Valdemarín, Entrambasaguas, Albarracín, Cuesta de la Cerroja, Cuesta del Pozuelo, Guadalaviar; "Grupo Oolítico")

Hinnites paniscus d'Orbigny (Valdecomadres, Virgen del Carmen, Bco. Hondo, Bco. Argudo, Pajares, Valdemarín, Entrambasaguas, Albarracín; "Grupo Oolítico")

Hinnites tenuistriatus d'Orbigny (Sarrión a Javalambre, Hoya de la Caridad; "Grupo Oolítico")

- Hinnites velatus Goldfuss (Valdecomadres, Virgen del Carmen, Bco. Hondo, Bco. Argudo, Pajares, Valdemarín, Entrambasaguas, Albarracín; "Grupo Oolítico")

Lima nov. sp. "vecina de la notata" (Calomarde; "Grupo Oolítico")

Lima obscura Sowerby (Hoya de la Caridad; "Grupo Oolítico")

- Lima proboscidea Sowerby (Cuesta de la Cerroja, Cuesta del Pozuelo y Guadalaviar; "Grupo Oolítico", "Liásico")

Lima tenuistriata Münster (Valdecomadres, Virgen del Carmen, Bco. Hondo, Bco. Argudo, Pajares, Valdemarín, Entrambasaguas, Albarracín; "Grupo Oolítico")

Mactromya aequalis Agassiz (Royuela; "Grupo Oolítico") Mytilus asper Sowerby (Cuesta de la Cerroja, Cuesta del Pozuelo y Guadalaviar; "Grupo Oolítico")

•Mytilus bipartitus Sowerby (Teruel; "Grupo Oolítico")

Mytilus pectinatus Sowerby (Cuesta de la Cerroja, Cuesta del Pozuelo, Guadalaviar Valdecomadres, Virgen del Carmen, Bco. Hondo, Bco. Argudo, Pajares, Valdemarín, Entrambasaguas, Albarracín; "Grupo Oolítico")

Mytilus plicatus Sowerby (Cuesta de la Cerroja, Cuesta del Pozuelo y Guadalaviar; "Grupo Oolítico")

Mytilus sowerbyanus d'Orbigny (Valdecomadres, Virgen del Carmen, Bco. Hondo, Bco. Argudo, Pajares, Valdemarín, Entrambasaguas, Albarracín; "Grupo Oolítico")

Ostrea amata d'Orbigny (entre Frías y Calomarde; Oxfordiense)

Ostrea arcata d'Orbigny (Teruel?; "Grupo Oolítico")

Ostrea colubrina Lamk. (Valdecomadres, Virgen del Carmen, Bco. Hondo, Bco. Argudo, Pajares, Valdemarín, Entrambasaguas, Albarracín; "Grupo Oolítico")

Pecten inaequicostatus Phillips (Valdecomadres, Virgen del Carmen, Bco. Hondo, Bco. Argudo, Pajares, Valdemarín, Entrambasaguas, Albarracín, Teruel; "Grupo Oolítico")

Pecten barbatus Sowerby (Valdecomadres, Virgen del Carmen, Bco. Hondo, Bco. Argudo, Pajares, Valdemarín, Entrambasaguas, Albarracín, Teruel; "Grupo Oolítico") Pecten lens Sowerby (entre Frías y Calomarde; Oxfordiense) Pholadomya acuticosta Sowerby (Monterde; "Grupo 
Oolítico")

Pleuromya arenacea Agassiz (Valdecomadres, Virgen del Carmen, Bco. Hondo, Bco. Argudo, Pajares, Valdemarín, Entrambasaguas, Albarracín; "Grupo Oolítico")

Pholadomya decussata Agassiz (entre Frías y Calomarde; Oxfordiense)

Pholadomya mariae d’Orbigny (Villar del Cobo; "Grupo Oolítico")

Pholadomya murchisoni Sowerby (Royuela, Valdecomadres, Virgen del Carmen, Bco. Hondo, Bco. Argudo, Pajares, Valdemarín, Entrambasaguas, Albarracín, Cuesta de la Cerroja, Cuesta del Pozuelo, Guadalaviar, Royuela, entre Peracense y Villafranca del Campo, Hoya de la Caridad, Sarrión a Javalambre, Sierra de Camarena, Josa a Hoz de la Vieja; "Grupo Oolítico")

Pholadomya ouralensis d'Orbigny (entre Frías y Calomarde; Oxfordiense)

- Pholadomya protei Defrance (Río Arcos; "Grupo Oolítico")

Pinnigena saussurei d'Orbigny (Pobo; "Grupo Oolítico")

Bivalvos del Jurásico Superior mencionados por Dereims (1898). - Especies identificadas en este trabajo:

- Ceromya excentrica Agassiz (Sierra de Javalambre; Jurásico Superior)

Cucullaea concinna Quenstedt (Sierra de Albarracín; Jurásico Superior)

Lima quenstedti Moesch (Sierra de Albarracín; Jurásico Superior)

Mactromya rugosa Agassiz (Sierra de Albarracín; Jurásico Superior)

Mytilus subpectinatus (Herrera-Villar de los Navarros; Jurásico Superior)

Ostrea bruntrutana (Castellón; Kimmeridgiense)

Ostrea cf. matronensis de Loriol (Sierra de Albarracín; Jurásico Superior)

Ostrea flabellata (Sierra de Albarracín, Sierra Palomera, Sima de San Pedro; Jurásico Superior)

Ostrea virgula (Castellón; Kimmeridgiense)
Ostrea cf. bruntrutana Thurmann (Sierra de Albarracín; Jurásico Superior)

- Pholadomya acuminata Hartmann (Jabaloyas-Griegos; Oxfordiense inferior)

Pholadomya cf. protei Brongniart (Sierra de Albarracín; Jurásico Superior)

- Pholadomya protei Defrance (Sierra de Javalambre; Jurásico Superior)

Pinnigena saussurei d'Orbigny (Sierra de Javalambre; Jurásico Superior)

Bivalvos del Jurásico mencionados por Riba (1959). -Especies identificadas en la Rama Aragonesa de la Cordillera Ibérica:

Alectryonia sp. (Sierra de Albarracín, Villar del Cobo; Lías superior, Jurásico Medio y Superior)

Arctostrea eruca Rollier (Griegos; Jurásico Medio y Superior)

- Ctenostreon proboscideum (?) Sowerby (Griegos; Jurásico Medio y Superior)

Ctenostreon sp. (Villar del Cobo, Frías de Albarracín; Lías superior, Jurásico Medio y Superior)

Goniomya v-scripta Sowerby (Frías de Albarracín; Jurásico Medio y Superior)

- Hinnites velatus d'Orbigny (Jabaloyas; Jurásico Medio y Superior)

Homomya sp. (Jabaloyas; Jurásico Medio y Superior)

Ostrea cf. bruntrutrana Thurmann (Jabaloyas; Kimmeridgiense (?))

Ostrea cf. matronensis de Loriol (Jabaloyas; Kimmeridgiense (?))

Ostrea sp. (Villar del Cobo, Albarracín-Gea, Frías de Albarracín, Jabaloyas; Liásico, Jurásico Medio y Superior) Pecten sp. (Villar del Cobo, Albarracín-Gea, Portillo de Tramacastilla, Frías de Albarracín, Jabaloyas; Liásico, Jurásico Medio y Superior)

Pholadomya sp. (Albarracín, Albarracín-Gea; Liásico)

Plagiostoma sp. (Entrambasaguas, Albarracín-Gea, Frías de Albarracín; Liásico, Jurásico Medio y Superior) 